\title{
Anti-hyphal formation property of allicin in suppression of Aspergillus fumigatus growth
}

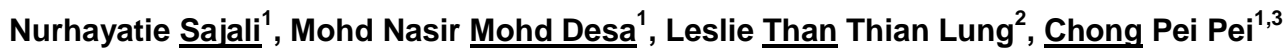 \\ ${ }^{1}$ Department of Biomedical Science, Faculty of Medicine and Health Sciences, Universiti Putra Malaysia, Serdang, \\ 43400 Selangor Darul Ehsan, Malaysia. \\ ${ }^{2}$ Department of Medical Microbiology and Parasitology, Faculty of Medicine and Health Sciences, Universiti Putra \\ Malaysia, Serdang, 43400 Selangor Darul Ehsan, Malaysia. \\ ${ }^{3}$ Institute of Bioscience, Universiti Putra Malaysia, Lebuh Silikon, Serdang, 43400 Selangor, Malaysia. \\ Email: cpp@medic.upm.edu.my
}

Received 5 March 2013; Received in revised form 18 March 2013; Accepted 23 March 2013

\begin{abstract}
Aims: The aim of this study was to examine whether allicin, a compound derived from fresh garlic, leads to growth inhibition and changes in the ultrastructure of the cell surface on medically important filamentous fungi, particularly Aspergillus fumigatus.

Methodology and results: The minimum inhibitory concentration (MIC) of allicin in A. fumigatus ATCC 36607 was determined by broth microdilution method according to the CLSI M38-A2 documents whereby the minimal fungicidal concentration (MFC) was determined by plating suspensions from visibly clear wells onto Sabouraud dextrose agar (SDA). Morphological changes on cell surface were observed through scanning electron microscopy (SEM) after $48 \mathrm{~h}$ incubation with allicin. In addition, time kill assay was conducted by incubating $A$. fumigatus at selected time points within $24 \mathrm{~h}$ period. Our finding indicated that the MIC and MFC for allicin were both $3.2 \mu \mathrm{g} / \mathrm{mL}$. Quantitative data for optical density obtained through microplate reader indicated that $p<0.05$ at MIC value in comparison with untreated control. Observation of allicin-treated cells through SEM demonstrated complete abrogation of hyphae formation at 3.2 $\mu \mathrm{g} / \mathrm{mL}$ and reduced mycelial growth at $1.6 \mu \mathrm{g} / \mathrm{mL}$ of allicin. This finding revealed anti-hyphal activity of allicin at 3.2 $\mu \mathrm{g} / \mathrm{mL}$. When $A$. fumigatus was incubated with $3.2 \mu \mathrm{g} / \mathrm{mL}$ allicin in the time course assay, the inhibitory effect of allicin was evident after $12 \mathrm{~h}$ incubation.

Conclusion, significance and impact of study: Our finding strongly implied that allicin exerts its antifungal activity against $A$. fumigatus via inhibiting the fungal cell proliferation as well as hindering transformation of the conidia into hyphae. Thus, this study depicted potential antifungal property of allicin to be used as alternative therapy to alleviate invasive fungal infection caused by $A$. fumigatus.
\end{abstract}

Keywords: Aspergillus fumigatus, allicin, minimum inhibitory concentration, minimum fungicidal concentration, time kill assay, scanning electron microscopy

\section{INTRODUCTION}

Allium sativum or commonly known as garlic has been recognised worldwide as a traditional medicine for treating various kinds of diseases. The historical perspective of its usage has been documented (Rivlin, 2001). As far as antimicrobial activities of garlic are concerned, Cavallito and Bailey had discovered as early as in 1944 that allicin or allyl 2-propene thiosulfinate, a pure compound from garlic possessed antibacterial activity (Cavallito and Bailey, 1944). Subsequently, other researchers had also found that allicin exhibited antifungal property (Yamada and Azuma, 1977; Shadkchan et al., 2004; Khodavandi et al., 2011), antiparasitic potential (Mirelman et al., 1987) and antimalarial activities (Coppi et al., 2006). It has been suggested that the antimicrobial properties of allicin are attributed to inhibition of sulfhydryl metabolic enzymes (Willis, 1956). Production of allicin involves a reaction between alliin which acts as a stable precursor with an enzyme called alliinase when garlic is crushed (Ellmore and Feldberg, 1994). Previous research has also reported several promising effects of allicin, not only in terms of its antimicrobial properties, but also protection against atherosclerosis onset (Lu et al., 2012).

Aspergillus fumigatus, a fungal mould characterised by mycelia formation, is the most common causative agent of invasive aspergillosis (Chakrabarti et al., 2011). Aspergillosis remains a critical illness among patients with debilitating immune systems (Dagenais and Keller, 2009). 
Patients with prolonged neutropenia, advance acquired immunodeficiency syndrome or AIDS, as well as those infected with chronic granulomatous disease and recipients of hematopoietic stem-cell transplants or solid organ transplantation are high risk groups of acquiring invasive aspergillosis (Segal and Walsh, 2006). Various commercial antifungal preparations have been widely used to treat aspergillosis. Amphotericin B has been regarded as a gold standard for treatment of invasive fungal infections since the 1960s (Ostrosky-Zeichner et al., 2003; Gibbs et al., 2005). However, the adverse effects of amphotericin B including various mechanisms of nephrotoxicity have been described; consequently, impede the usage of this drug for antifungal therapy (Laniado-Laborín and Cabrales-Vargas, 2009).

Although there is a major shift in polyene therapy, with amphotericin $B$ lipid preparations becoming the new "gold standard" in place of amphotericin B (OstroskyZeichner et al., 2003), a number of reasons have hindered the use of lipid formulations as a drug of choice. These include cost and scarcity of published safety data to guide clinicians (Kleinberg, 2006). Thus, these issues presented a strong justification to search for an alternative therapeutic candidate which has fewer side effects as a substitute for commercially prepared antifungal drugs. To date, there are less than five reports in the literature on the potential antifungal activity of allicin against $A$. fumigatus, and the mechanism through which allicin suppresses the growth of this pathogenic mould has not been investigated previously. In view of the antifungal properties of allicin, and the lack of study on allicin in its ability to interfere with the growth of $A$. fumigatus, this study was carried out to determine the minimum inhibitory concentration (MIC) and minimum fungicidal concentration (MFC) of allicin against $A$. fumigatus, and to observe the effects of allicin on the fungal cell surface changes through scanning electron microscopy (SEM).

\section{MATERIALS AND METHODS}

\section{Fungal strain}

A. fumigatus ATCC 36607 was obtained from American Type Culture Collection (ATCC). This strain was maintained on potato dextrose slant agar (PDA) and subcultured every 6 months to ensure viability.

\section{Antifungal agent}

Allicin was purchased from LKT laboratories, St Paul, USA (purity $\geq 98 \%$, cat no: LKT-A4440-M005) and allicin was dissolved at the concentration of $4 \mathrm{mg} / \mathrm{mL}$ in a solvent mixture of methanol, water and formic acid (60:40:1) as described previously (Khodavandi et al., 2011).

\section{Inoculum preparation}

Inoculum was prepared according to CLSI M38-A2 document (2008). A. fumigatus ATCC 36607 was grown on potato dextrose agar (Becton Dickinson, USA) for $48 \mathrm{~h}$ at $35^{\circ} \mathrm{C}$. Sporulating colonies were harvested by gently probing the colonies with pipette tips using a micropipette in the presence of $0.85 \%$ saline and three drops of Tween 20 (Nacalai Tesque, Japan) to facilitate the process. The resulting mixture of conidia and hyphal fragments were then transferred into a sterile tube and subsequently the density was adjusted at optical density $\left(O_{530 \mathrm{~nm}}\right)$ of 0.09 to 0.13 (T60 UV Visible Spectrophotometer, USA). A further 1:50 dilution was made in RPMI medium (SigmaAldrich, USA).

\section{Broth microdilution method}

About $0.1 \mathrm{~mL}$ of prepared allicin in a range of doubling concentration from $0.05-25.6 \mu \mathrm{g} / \mathrm{mL}$ was dispensed into a 96-well microtiter plate (Greiner Bio-One $\mathrm{GmbH}$, German) in ascending concentration. Subsequently, $0.1 \mathrm{~mL}$ of standardised inoculum were dispensed into wells containing the allicin with final concentration of 0.4 to $5 \times 10^{4} \mathrm{CFU} / \mathrm{mL}$ which makes the final concentration of allicin ranging from $0.025-12.8 \mu \mathrm{g} / \mathrm{mL}$. Growth control well contained standardised diluted inoculum and the drug diluents without allicin, whereas negative control contained only RPMI 1640 medium. The plate was incubated for $48 \mathrm{~h}$ at $35{ }^{\circ} \mathrm{C}$. MIC was determined with unaided eye and quantitative data was reported spectrophotometrically at $\mathrm{OD}_{530 \mathrm{~nm}}$ using microplate reader (Dynex Technologies, USA). The percentage of growth inhibition at MIC was determined using the formula (Patton et al., 2006): Percent of inhibition $=1-(O D$ test well $/$ OD untreated control well) $\times 100$.

\section{Determination of minimum fungicidal concentration (MFC)}

Fungicidal activity of allicin on $A$. fumigatus was determined according to the method described by EspinelIngroff (1998), with slight modification. Twenty microlitres of aliquots were pipetted from optically clear wells that exhibited complete inhibition (growth similar to growth control well) and from growth control well. These suspensions were subcultured onto Sabouraud dextrose agar plates. The plates were incubated for 2 days at $35^{\circ} \mathrm{C}$ or until growth was observed on growth control plates. The lowest drug concentration that demonstrated in either no growth or fewer than three colonies which inferred $99.9 \%$ of killing was term as MFC (Espinel-Ingroff, 1998).

\section{Scanning electron microscopy}

A. fumigatus ATCC36607 inoculum was prepared as described previously (CLSI, 2008) with modifications in the total volume. One millilitre of standardised inoculum was treated with $1 \mathrm{~mL}$ of $1 / 4 \times \mathrm{MIC}, 1 / 2 \times \mathrm{MIC}, 1 \times \mathrm{MIC}$ and $2 \times$ MIC of allicin respectively. Untreated inoculum served as a growth control. Samples were incubated at $35^{\circ} \mathrm{C}$ for $48 \mathrm{~h}$. Each sample was placed into separate vials and fixed with $4 \%$ glutaraldehyde (Agar Scientific, UK) for $4 \mathrm{~h}$ at $4{ }^{\circ} \mathrm{C}$. Samples were rinsed with $0.1 \mathrm{M}$ sodium 
cacodylate buffer for 3 times, 10 min each. The samples were post-fixed in $1 \%$ osmium tetroxide Table 1: Effect of allicin on A. fumigatus ATCC 36607 based on OD differences and its percentage of inhibition at MIC calculated normalize to growth control. Results are reported as means of three independent experiments done in triplicate.

\begin{tabular}{|c|c|c|c|c|c|}
\hline \multirow{2}{*}{ Fungal strain } & \multicolumn{3}{|c|}{ OD } & \multirow{2}{*}{$\begin{array}{l}\text { Percentage of } \\
\text { inhibition (\%) }\end{array}$} & \multirow{2}{*}{$\begin{array}{c}\text { Minimum } \\
\text { fungicidal } \\
\text { concentration }\end{array}$} \\
\hline & Growth control $^{\mathrm{a}}$ & Control $^{b}$ & $\begin{array}{c}\text { Allicin }^{\mathrm{c}} \\
\text { (3.2 mg/L) }\end{array}$ & & \\
\hline $\begin{array}{l}\text { A. fumigatus } \\
\text { ATCC36607 }\end{array}$ & $0.349 \pm 0.058$ & $0.086 \pm 0.001$ & $\begin{array}{c}0.079 \pm 0.001^{d} \\
(p=0.046)\end{array}$ & $77.4 \%{ }^{\mathrm{e}}$ & $3.2 \mu \mathrm{g} / \mathrm{mL}$ \\
\hline $\begin{array}{l}\text { nngus+broth } \\
\text { oth only } \\
\text { eated with allicin } \\
\text { ean } \pm S E M \text { is sign } \\
\text { ercentage of inhil }\end{array}$ & $\begin{array}{l}\text { MIC } \\
\text { thly different }(p<0 \\
\text { at } \mathrm{MIC}=[1-(0.0)\end{array}$ & $\begin{array}{l}3.2 \mu \mathrm{g} / \mathrm{mL} \text { allic } \\
49)] \times 100\end{array}$ & d cells in com & vith growth c & \\
\hline
\end{tabular}

(Agar Scientific, UK) for $2 \mathrm{~h}$ at $4{ }^{\circ} \mathrm{C}$. Samples were rinsed again with $0.1 \mathrm{M}$ sodium cacodylate buffer followed by dehydrating in a series of different concentrations of alcohol starting with $20 \%$ until $100 \%$ (v/v) (ChemAR ${ }^{\circledR}$ Systerm, Malaysia) and finally with $100 \%$ (v/v) acetone (Merck, USA). Samples were transferred into specimen basket and put into critical point dryer (Bal-tec, Germany) for $30 \mathrm{~min}$. Coverslips with samples attached to it were then adhered onto the stub and coated with gold in sputter coater (Bal-tec, Germany) prior to viewing under SEM (LEO 1455 VPSEM) attached with energy dispersive $X$ ray (EDX).

\section{Time kill assay}

Effect of allicin towards A. fumigatus within $24 \mathrm{~h}$ was observed through time kill assay following the method described by Shadkchan et al., (2004), with slight modifications. A. fumigatus ATCC36607 was grown in the presence of $3.2 \mu \mathrm{g} / \mathrm{mL}$ allicin. This concentration was chosen because it was the concentration that inhibits the growth of $A$. fumigatus as determined by broth microdilution method (CLSI, 2008). Conidia was serially diluted in tenfold dilution after $0,2,4,6,8,10,12$ and $24 \mathrm{~h}$ incubation before plated onto Sabouraud dextrose agar. The plates were incubated for $48 \mathrm{~h}$ at $35^{\circ} \mathrm{C}$. Colonies were counted and compared with untreated control at $0 \mathrm{~h}$. Percentage of survival was plotted against incubation with allicin using GraphPad Prism Version 5.02.

\section{Statistical analysis}

Each experiment was performed in triplicate. Values were expressed as mean \pm standard error of mean (SEM) and mean difference for each OD reading for the respective concentrations were compared with growth control by performing Mann-Whitney $U$ for two independent samples test, using Statistical Package for the Social Sciences (SPSS) version 16.0 with $p<0.05$ deemed as statistically significant.

\section{RESULTS AND DISCUSSION}

\section{Antifungal susceptibility testing and minimum fungicidal concentration}

The minimum inhibitory concentration of allicin was determined to be $3.2 \mu \mathrm{g} / \mathrm{mL}$ against $A$. fumigatus ATCC 36607 , as observed visually. This was further supported by quantitative values which were based on OD reading. In comparison with growth control which did not contained allicin, there was a significant difference $(p<0.05)$ in the OD reading in fungal cells treated with allicin at MIC concentration as shown in Table 1. At this concentration, percentage of inhibition was $77.4 \%$. However, the MFC was also found to be $3.2 \mu \mathrm{g} / \mathrm{mL}$.

Previous study by Shadkchan et al., (2004) reported both MIC and MFC for allicin on $A$. fumigatus was determined to be $8 \mu \mathrm{g} / \mathrm{mL}$. Differences in MIC and MFC values between previous study and present study could be due to variation in the allicin preparations used in both studies which involved different synthesis and purification procedures. Indeed, various methods of synthesizing allicin had been reported previously, and this has contributed to differing grades and purities of allicin obtained (Dušica et al., 2011). Several techniques of allicin synthesis involve oxidation of allyl sulphide by hydrogen peroxide in acid medium by ESR spin trap method (Nikolić et al., 2004) and processing of dichloromethane solution of allyl disulfide by magnesium monoperoxy hydrate in the presence of ammonium-butyl sulphate (Cruz-Villalon, 2001).

Additionally, variation in the assay methods in determining the purity of the compound could be one of the key factors for the varying activities of allicin reported in different studies. In our study, the purity of allicin was $\geq$ $98 \%$ according to the manufacturer, which was assayed using UPLC (ultra performance liquid chromatography). In contrast, previous studies described that their source of allicin was produced by a reaction between the synthetic 
substrate of alliin with allinase through an immobilised allinase column and assayed using high performance liquid chromatography (HPLC). UPLC provides significant advances compared to conventional HPLC due to its rapid operation time, higher sensitivity and resolution (Srivastava et al., 2010).

\section{Morphological changes}

Apart from the antifungal susceptibility data obtained, the effect of allicin against $A$. fumigatus ATCC 36607 was also examined morphologically through SEM at $1 / 4 \times \mathrm{MIC}$, $1 / 2 \times$ MIC, $1 \times$ MIC and $2 \times$ MIC. Perturbation to the cell surface morphology could be seen when $A$. fumigatus ATCC 36607 was treated with various concentrations of allicin. Scanning electron microscopy images captured at 10,000X and 1000X magnification exhibited significant changes in treated $A$. fumigatus hyphae and conidial morphology compared with growth control.

Untreated growth control of $A$. fumigatus was characterised by smooth cell surface of septate hyphae with a few branches and linear, elongated mycelia with apparently consistent diameter throughout the length of the hyphae as shown in Figures 1 (a) and 2 (a). However, allicin-treated $A$. fumigatus in Figure 1 (b) exhibited uneven and rough surface which denote the effect of treatment at $1 / 4 \times$ MIC. In marked contrast, allicin-treated $A$. fumigatus in Figure 1 (c) shows irregular shape of hyphae with irregular constriction and bulging along the length of the hyphae. In addition, the cell surface of hyphae was observed to be crinkled, patchy and uneven in which it might indicate possible cell wall or cell membrane disruption when $A$. fumigatus was treated with allicin at this concentration $(1 / 2 \times \mathrm{MIC})$. When examined under lower magnification at 1000X, fewer mycelia were observed as shown in Figures 2 (b) and 2 (c), with distorted and wavy shape at certain areas of the hyphae. Complete inhibition of hyphal growth was observed in $A$ fumigatus when treated with both $1 \times \mathrm{MIC}$ and $2 \times \mathrm{MIC}$ as illustrated in Figures 1(d), (e) and Figure 2 (d), (e). Complete disruption in morphological of conidia could be seen in Figures $1(\mathrm{e})$ and 2 (e).

Scanning electron microscopy observation indicated that treatment of $A$. fumigatus at $3.2 \mu \mathrm{g} / \mathrm{mL}(1 \times \mathrm{MIC})$ and $6.4 \mu \mathrm{g} / \mathrm{mL}(2 \times \mathrm{MIC})$ of allicin has impeded the transformation of hyphae from conidia. This could be due to anti-hyphal properties of allicin at these concentrations, which subsequently led to complete absence of formation of hyphae. Hyphae formation and penetration into host tissue are primarily associated with pathogenesis of invasive aspergillosis. When conidia are being inhaled by immunocompromised patients or those with weakened immune system, the conidia will initially colonise and invade pulmonary epithelium, which will eventually germinate into invasive hyphae. Subsequently, angioinvasion takes place in which hyphae penetrate blood capillaries. Simultaneous dissemination of hyphal fragment as well as other toxic molecule finally leads to dissemination in deep organ (Askew, 2008; Abad et al., 2010). Hence, suppression of hyphae formation by allicin highlights the potent anti-hyphae activity of this compound and thus underlies its potential use for preventing the development of organ-invasive aspergillosis. It would be interesting to investigate whether allicin could exhibit a similar degree of anti-hyphae property against $A$. fumigatus in an in vivo model utilising a mouse model of invasive aspergillosis.

In relation to this finding, Yoshida et al. (1987) has reported the effect of ajoene, one of the bioactive compounds derived from garlic on the ultrastructure of Aspergillus niger. Treatment with ajoene at the inhibitory concentration of $20 \mu \mathrm{g} / \mathrm{mL}$ demonstrated surface depression or flat ribbon-like structure. Our present study found that allicin at a lower inhibitory concentration than that reported for ajoene, which is $3.2 \mu \mathrm{g} / \mathrm{mL}$, was able to exert complete inhibition of hyphae formation in $A$. fumigatus. Hence, this finding underscores the potent activity of allicin on the filamentous fungus. In this study, the irregular shape of hyphae with uneven surface featuring constriction and bulging appearance along the hyphae were clearly seen in $A$. fumigatus treated with $1 / 4 \times$ MIC and $1 / 2 \times$ MIC. However, the underlying mechanism through which allicin acts to cause these morphological changes is still unclear. It could possibly be due to a disruption in the homeostasis process which leads to a defect in hyphae morphology, or it could also be attributed to the ability of allicin to target a hypha-specific cell wall components or inhibition of sulfhydryl metabolic enzymes as previously reported (Willis, 1956).

In addition, the effects of micafungin, a commercially prepared antifungal drug on $A$. fumigatus have been described by Nishiyama et al. (2005). Treatment of $A$. fumigatus with various concentrations of micafungin ranging from $0.001-0.1 \mu \mathrm{g} / \mathrm{mL}$, within $2-5 \mathrm{~h}$ incubation period has contributed to changes in hyphal morphology. Short branches, with rough, wrinkled surface and subsequently disruption of hyphae were observed at various concentrations of treatment within the incubation period. Although the study demonstrated that lower micafungin concentration resulted in defects in hyphal morphology, direct suppression of hyphae formation by this drug against $A$. fumigatus was not unambiguously portrayed. This could be due to the nature of the experiment that was being carried out after most of the conidia had germinated into hyphae. Germination of conidia into hyphae is the main culprit in pathogenesis of invasive aspergillosis and hence treatment should be done before the germination process occur. Thus, the previous finding was dissimilar with our study which pinpoints the effect of allicin on conidia treated with allicin before they had germinated into hyphae.

\section{Time kill assay}

After $10 \mathrm{~h}$ incubation with allicin, the growth of $A$. fumigatus ATCC36607 had decreased to half as shown in the time kill curve in Figure 3, and the growth diminished gradually after $12 \mathrm{~h}$ and began to plateau after $24 \mathrm{~h}$. However, there was a small fluctuation in the decreasing trend of growth within the first $12 \mathrm{~h}$ of incubation. 
Phenotypically, colonies that survived after the cells had

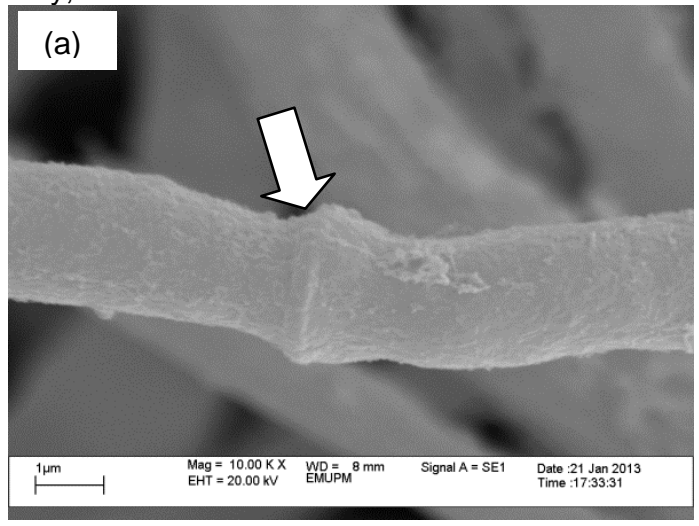

been treated with allicin appeared smaller in contrast with
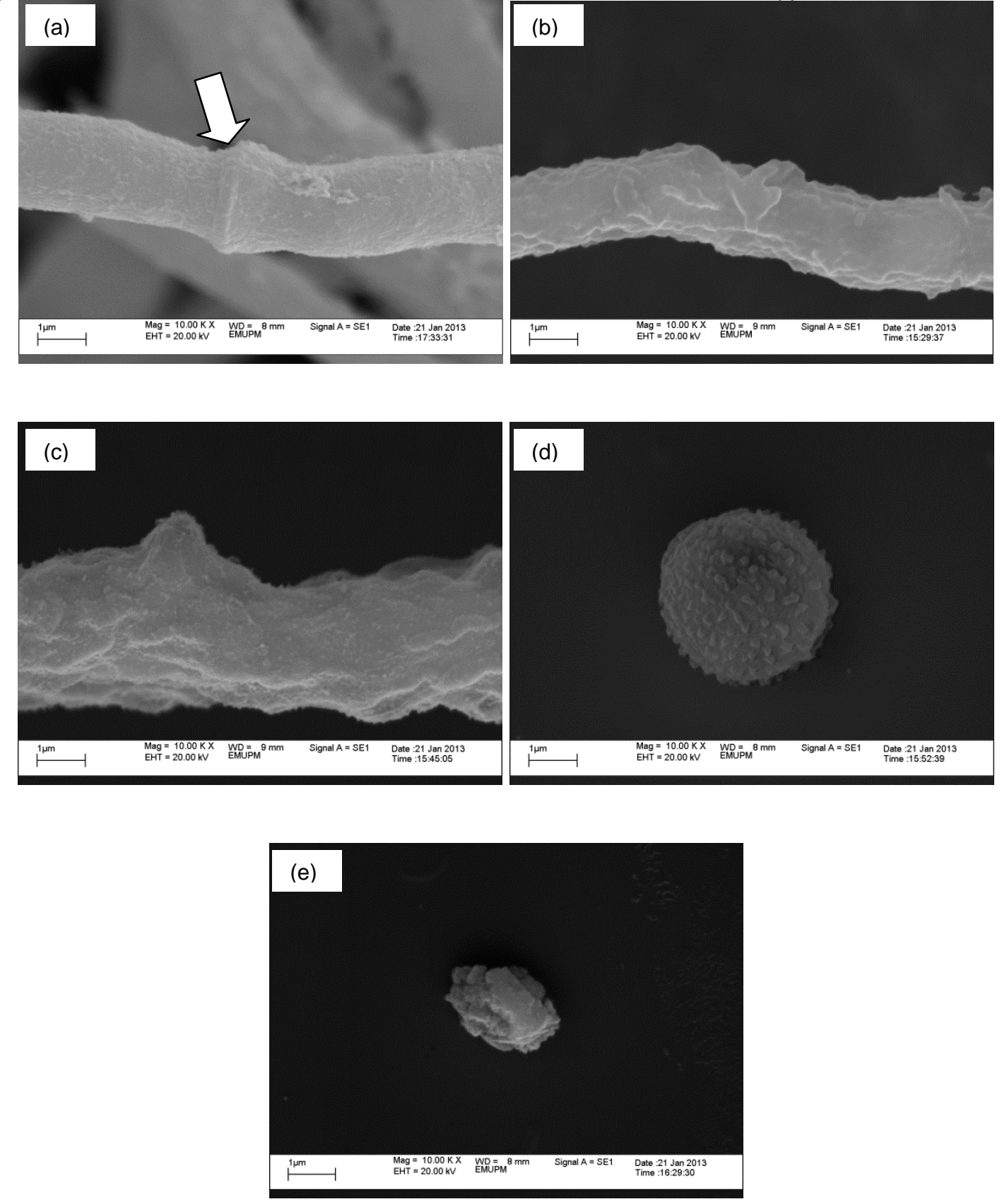

Figure 1: Scanning electron micrographs of $A$. fumigatus surface ultrastucture observed after $48 \mathrm{~h}$ incubation with allicin. (a) Growth control, (b) A. fumigatus treated at $1 / 4 \times$ MIC, (c) $1 / 2 \times$ MIC and (d) $1 \times$ MIC (e) $2 \times$ MIC. Magnification $\times$ 10,000 . Septate hyphae can be observed in growth control as indicated by an arrow symbol.

colonies in untreated control which appeared larger and were clumped together (data not shown). Colony counting was feasible post-treatment with allicin but not for the untreated control at the extended time points of growth due to the formation of clustered colonies.
On contrary, Shadkchan et al. (2004) reported that $A$. fumigatus started to display reduction in growth after $8 \mathrm{~h}$ incubation. This discrepancy in both findings could be due to the difference in fungicidal concentration of allicin 
treated upon $A$. fumigatus in which we observed the effect

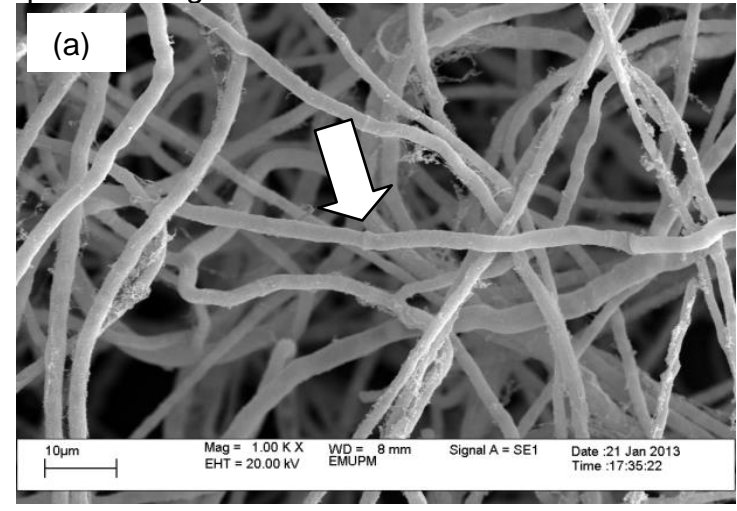

of $1 \times$ MIC in present study whereas the previous study
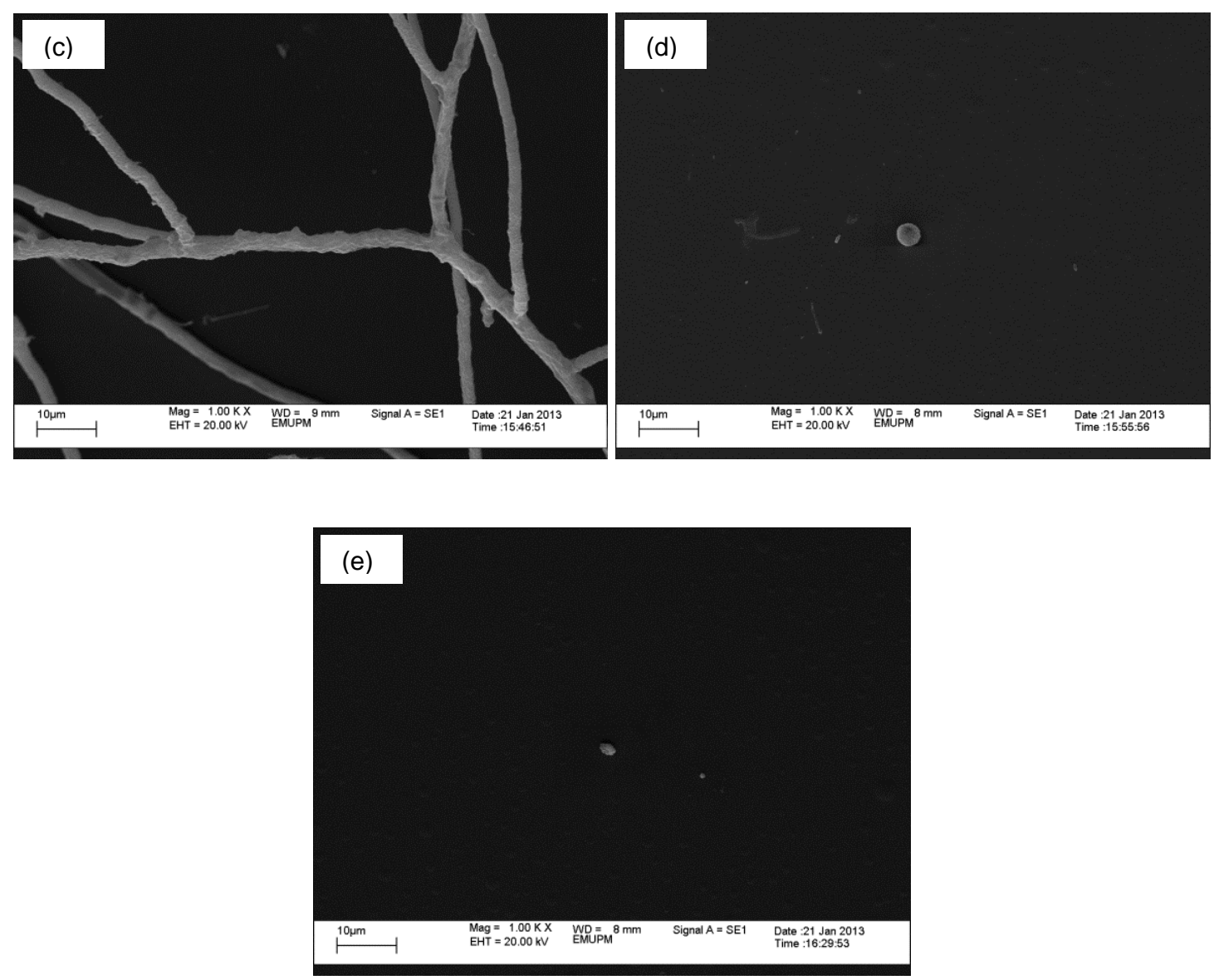

Figure 2: Scanning electron micrographs of $A$. fumigatus surface ultrastucture observed after $48 \mathrm{~h}$ incubation with allicin. (a) Growth control, (b) A. fumigatus treated at $1 / 4 \times$ MIC, (c) $1 / 2 \times$ MIC and (d) $1 \times$ MIC (e) $2 \times$ MIC. Magnification $\times$ 1000. Septate hyphae can be observed in growth control as indicated by an arrow symbol. 


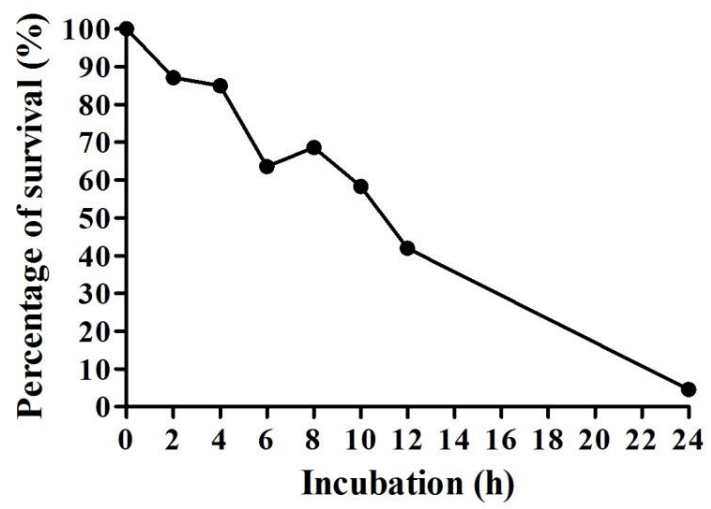

Figure 3: Percentage of $A$. fumigatus ATCC 36607 survival (\%) against incubation time with allicin (h). Results are representative of three independent experiments performed in triplicate. Growth percentages were calculated by dividing average colonies counted for each selected time point with untreated control at $0 \mathrm{~h}$.

examined the allicin concentration at two-fold above MFC which is $2 \times$ MFC. Taken together, the findings from both studies denote that a higher concentration of allicin decreases the time taken for the fungicidal effect to be exhibited. In time kill assay, time exposure and ability of conidia to be permeated and subsequently eradicated by antifungal compound play a part in the fungicidal activity (Hammer et al., 2002).

\section{CONCLUSION}

In conclusion, the anti-hyphal and growth inhibitory properties of allicin against $A$. fumigatus could be observed through broth microdilution test, SEM and time kill assay. More studies need to be done to establish the efficacious range of concentration for allicin in vivo and to rule out any possible toxicity to humans prior to any human clinical trial before it can be developed as an antifungal agent on par with commercial antifungal drugs such as amphotericin B and fluconazole. On the other hand, further fundamental investigations could include ultrastructural observation of internal components of cells under transmission electron microscopy which would denote changes in cytosolic components, cell membrane and cell wall structure post-treatment with allicin. In addition, elucidation of the mechanism of action of allicin through quantifying the expression level of targeted genes that are implicated in hyphae-specific cell well related genes could further bolster the premise of allicin as an alternative antifungal agent.

\section{ACKNOWLEDGEMENT}

This study was supported by a research grant of the Universiti Putra Malaysia, RUGS grant Initiative 6 (Project number: No. 04-01-11-1167RU).

\section{REFERENCES}

Abad, A., Fernández-Molina, J. V., Bikandi, J., Ramírez, A., Margareto, J., Sendino, J., Hernando,
F. L., Pontón, J., Garaizar, J. and Rementeria, A. (2010). What makes Aspergillus fumigatus a successful pathogen? Genes and molecules involved in invasive aspergillosis. Revista Iberoamericana de Micología 27, 155-182.

Askew, D. S. (2008). Aspergillus fumigatus: Virulence genes in a street-smart mold. Current Opinion in Microbiology 11, 331-337.

Cavallito, C. and Bailey, J. H. (1944). Allicin, the antibacterial principle of Allium sativum. Isolation, physical properties and antibacterial action. Journal of the American Chemical Society 66, 1950-1951.

Chakrabarti, A., Chatterjee, S. S., Das, A. and Shivaprakash, M. R. (2011). Invasive aspergillosis in developing countries. Medical Mycology 49, S35-S47.

CLSI. (2008). Reference Method for Broth Dilution Antifungal Susceptibility Testing of Filamentous Fungi; Approved Standard-Second Edition. CLSI document M38-A2. Clinical and Laboratory Standards Institute, Wayne, PA.

Coppi, A., Cabinian, M., Mirelman, D. and Sinnis, P. (2006). Antimalarial activity of allicin, a biologically active compound from garlic cloves. Antimicrobial Agents and Chemotherapy 50, 731-737.

Cruz-Villalon, G. (2001). Synthesis of allicin and purification by solid-phase extraction. Analytical Biochemistry 290, 376-378.

Dagenais, T. R. T. and Keller, N. P. (2009). Pathogenesis of Aspergillus fumigatus invasive aspergillosis. Clinical Microbiol Review 22, 447-465.

Dušica, P. I., Vesna, D. N., Ljubiša, B. N., Mihajlo, Z. S., Ljiljana, P. S. and Milorad, D. C. (2011). Allicin and related compounds: biosynthesis, synthesis and pharmacological activity. Facta Universitatis - series: Physics, Chemistry and Technolog. 9, 9-20.

Ellmore, G. and Feldberg, R. (1994). Alliin lyase localization in bundle sheaths of garlic clove (Allium sativum). American Journal of Botany 81, 89-95.

Espinel-Ingroff, A. (1998). Comparison of in vitro activities of the new triazole SCH56592 and the echinocandins MK-0991 (L-743, 872) and LY303366 
against opportunistic filamentous and dimorphic fungi and yeasts. Journal of Clinical Microbiology 36, 29502956.

Gibbs, W. J., Drew, R. H. and Perfect, J. R. (2005). Liposomal amphotericin B: clinical experience and perspectives. Expert Review of Anti- Infective Therapy 3, 167-181.

Hammer, K. A., Carson, C. F. and Riley, T. V. (2002). In vitro activity of Melaleuca alternifolia (tea tree) oil against dermatophytes and other filamentous fungi. Journal of Antimicrobial Chemotherapy 50, 195-199.

Khodavandi, A., Alizadeh, F., Harmal, N. S., Sidik, S. M., Othman, F., Sekawi, Z., Jahromi, M. A. F., Ng, K.-P. and Chong, P. P. (2011). Comparison between the efficacy of allicin and fluconazole against Candida albicans in vitro and in a systemic candidiasis mouse model. FEMS Microbiology Letters 315, 87-93.

Kleinberg, M. (2006). What is the current and future status of conventional amphotericin B? International Journal of Antimicrobial Agents 27S, S12-S16.

Laniado-Laborín, R. and Cabrales-Vargas, M. N. (2009). Amphotericin B: side effects and toxicity. Revista lberoamericana de Micología 26, 223-227.

Lu, Y., He, Z., Shen, X., Xu, X., Fan, J., Wu, S. and Zhang, D. (2012). Cholesterol-lowering effect of allicin on hypercholesterolemic ICR mice. Oxidative Medicine and Cellular Longevit. 2012, 1-6.

Mirelman, D., Monheit, D. and Varon, S. (1987). Inhibition of growth of Entamoeba histolytica by Allicin, the active principle of garlic extracts (Allium sativum). Journal of Infectious Diseases 156, 243244.

Nikolić, V., Stanković, M., Nikolić, L. J. and Cvetković, D. (2004). Mechanism and kinetics of synthesis of allicin. Pharmazie 59, 10-14.

Nishiyama, Y., Hasumi, Y., Ueda, K., Uchida, K. and Yamaguchi, H. (2005). Effects of micafungin on the morphology of Aspergillus fumigatus. Journal of Electron Microscopy (Tokyo) 54, 67-77.

Ostrosky-Zeichner, L., Marr, K. A., Rex, J. H. and Cohen, S. H. (2003). Amphotericin B: Time for a new "Gold Standard". Clinical Infectious Diseases 37, 415425.

Patton, T., Barrett, J., Brennan, J. and Moran, N. (2006). Use of a spectrophotometric bioassay for determination of microbial sensitivity to manuka honey. Journal of Microbiological Methods 64, 84-95.

Rivlin, R. S. (2001). Historical perspective on the use of garlic. Journal of Nutrition 131, 951S-954S.

Segal, B. H. and Walsh, T. J. (2006). Current approaches to diagnosis and treatment of invasive aspergillosis. American Journal of Respiratory and Critical Care Medicine 173, 707-717.

Shadkchan, Y., Shemesh, E., Mirelmen, D., Miron, T., Rabinkov, A., Wilchek, M. and Osherov, N. (2004). Efficacy of allicin, the reactive molecule of garlic, in inhibiting Aspergillus spp. in vitro, and in a murine model of disseminated aspergillosis. Journal of Antimicrobial Chemotherapy 53, 832-836.
Srivastava, B., Sharma, B. K., Baghel, U. S. and Yashwant, S. N. (2010). Ultra performance liquid chromatography (UPLC): A chromatography technique. International Journal of Pharmaceutical Quality Assurance 2, 19-25.

Willis, E. (1956). Enzyme inhibition by allicin, the active principle of garlic. Biochemical Journal 63, 514-520.

Yamada, Y. and Azuma, K. (1977). Evaluation of the in vitro antifungal activity of allicin. Antimicrobial Agents and Chemotherapy 11, 743-749.

Yoshida, S., Kasuga, S., Hayashi, N., Ushiroguchi, T., Matsuura, H. and Nakagawa, S. (1987). Antifungal activity of ajoene derived from garlic. Applied and Environmental Microbiology 53, 615-617. 